\title{
Comportamiento a la Corrosión del Acero API X70 Soldado por el Proceso de Doble Arco Sumergido Inmerso en Diferentes Medios Corrosivos
}

\author{
Omar Francisco Montes ${ }^{1}$, Rocío Saldaña Garcés², Felipe A. Reyes Reyes ${ }^{1}$, Patricia del Carmen Zambrano Robledo ${ }^{3}$, \\ Francisco H. Estupiñán López ${ }^{3}$, Facundo Almeraya Calderón ${ }^{3}$ \\ 1 Corporación Mexicana de Investigación en Materiales, S.A. de C.V. - COMIMSA, Departamento de Estudios de Posgrado en \\ Tecnología de la Soldadura Industrial, Saltillo, Coahuila, México. \\ 2 Catedrática Consejo Nacional de Ciencia y Tecnologia - CONACYT, Corporación Mexicana de Investigación en Materiales, \\ S.A. de C.V. - COMIMSA, Saltillo, Coahuila México. \\ 3 Universidad Autónoma de Nuevo León - UANL, Centro de Investigación e Innovación en Ingeniería Aeronáutica - CIIIA, \\ Facultad de Ingeniería Mecánica y Eléctrica - FIME, Carretera a Salinas Victoria, Apodaca, Nuevo León, México.
}

Recibido: 28 Enero, 2016

Aprobado: 01 Jun., 2016

E-mails: omontes@alumnos-comimsa.mx (OFM), areyes@comimsa.com (FARR), rocio.saldana@comimsa.com (RSG), patricia.zambranor@uanl.mx (PCZR), festupinan.ciiia@gmail.com (FHEL), falmeraya.uanl.ciiia@gmail.com (FAC)
Resumen: Los aceros API X70 son aplicados en fabricación y construcción de tuberías para el transporte de hidrocarburos, sin embargo, a un lapso de tiempo se degradan debido al medio ambiente al cual están expuestos; influyendo en la velocidad y tipo de corrosión sobre las superficies del metal base (MB), zona afectada por el calor (ZAC) y zona de fusión (ZF). A fin de conocer y evaluar la susceptibilidad a la corrosión en uniones del acero API X70 sometido a diferentes medios corrosivos, varias pruebas se llevaron a cabo por el proceso de soldadura de doble arco sumergido (DSAW, por sus siglas en inglés), las cuales fueron inmersas en diferentes electrolitos: Cloruro de Sodio $\left(\mathrm{NaCl}\right.$ al 3,5\%) y Agua $\left(\mathrm{H}_{2} \mathrm{O}\right)$ a temperatura ambiente para conocer su comportamiento. Se utilizaron técnicas electroquímicas de ruido electroquímico (RE) y curvas de polarización cíclica (CPC) para obtener velocidad y tipo de corrosión producida en cada una de las muestras analizadas, incluyendo las tres zonas: MB, ZAC y ZF. Además, se utilizaron técnicas de caracterización como microscopía óptica (MO) y microscopía electrónica de barrido (MEB) para el análisis superficial. La mayoría de las soldaduras presentan corrosión mixta, con mayores velocidades de corrosión en 3,5\% de $\mathrm{NaCl}$.

Palabras-clave: Corrosión; Técnicas electroquímicas; Soldadura de doble arco sumergido.

\section{Corrosion Behavior of API X70 Steel Welded for Process Double Submerged Arc Immersed in Different Corrosives Environments}

\begin{abstract}
The steels API X70 are applied in construction and fabrication of pipeline for transport of hydrocarbons, however, to lapse time are degrade due to environment to which are exposed, influencing on mode and corrosion rate on surface base metal (BM), heat zone affected (HAZ) and fusion zone (FZ). In order to know and evaluate the corrosion susceptibility in joints of API X70 steel submitted to different corrosives environment, several tests were carried out by process of double submerged arc welding (DSAW), which were immersed in different electrolytes: Sodium Chloride $(3.5 \% \mathrm{NaCl})$ and Water $\left(\mathrm{H}_{2} \mathrm{O}\right)$ at room temperature for know its behavior. Electrochemical techniques were used of electrochemical noise (EN) and cyclic polarization curves (CPC) to obtain mode and corrosion rate produced for each of the samples analyzed, included the three zones: BM, HAZ and FZ. Further, characterization techniques were used such as optical microscopy (OM) and scanning electron microscopy (SEM) for superficial analysis. The welds' majoirity have a mixed corrosion, with higher corrosion rate in $3.5 \% \mathrm{NaCl}$.
\end{abstract}

Key-words: Corrosion; Electrochemical techniques; Double submerged arc welding.

\section{Introducción}

Actualmente en la industria del petróleo, el empleo de materiales que sean capaces de satisfacer las necesidades en este sector son cada vez más rigurosos debido a las condiciones de operación a los cuales se encuentran expuestos; demandan el uso de metales que sean capaces de cumplir con la calidad suficiente para el desempeño correcto.

Los aceros API 5L son considerados como HSLA (High Strenght Low Alloy) los cuales contienen elementos como niobio, vanadio y titanio; estos elementos son conocidos 
Comportamiento a la Corrosión del Acero API X70 Soldado por el Proceso de Doble Arco Sumergido Inmerso en Diferentes Medios Corrosivos

como microalentes por las pequeñas cantidades requeridas cuya finalidad es mejorar las propiedades mecánicas del material $[1,2,3]$.

El uso de procesos de soldadura por doble arco sumergido consiste en realizar una doble costura sobre los materiales tanto en la superficie interna como en la externa de las tuberías [4]. En la actualidad se han utilizado los aceros API 5L X70 y el proceso DSAW para fabricar tuberías empleadas para conducción de hidrocarburos y gases; por las excelentes propiedades mecánicas y soldabilidad del acero [1], la obtención de productos de alta calidad y costos razonables además de producir soldaduras de excelente penetración, completa fusión de los materiales y por consiguiente una mayor resistencia [5,6]; sin embargo uno de los principales inconvenientes que se han presentado con el lapso del tiempo en los aceros para tuberías de línea durante el servicio, es la exposición a soluciones y ambientes corrosivos que generan un daño irreversible y catastrófico a través de la degradación del material [7].

Mediante las técnicas electroquímicas es factible determinar el comportamiento de la corrosión electroquímica en los materiales como velocidades de corrosión, potenciales y corrientes de corrosión, tipos de corrosión [8,9]. Varios investigadores se han enfocado en estudios para evaluar el comportamiento a la corrosión electroquímica de aceros HSLA, inoxidables, hierros a través de las técnicas de ruido electroquímico y curvas de polarización y los cuales son expuestos a medios salinos con determinadas concentraciones y diferentes intervalos de tiempo de exposición. Analizando los efectos en las microestructuras, la susceptibilidad a las picaduras en medios corrosivos [7,10-12]. Estudios comparativos entre materiales similares al de este estudio y otros, sobre el comportamiento a la corrosión como son el hierro puro y el acero API 5 L X65 expuestos en soluciones de $4 \%$ de $\mathrm{NaCl}$ en intervalos de exposición de 48 minutos y 12 horas, establecen que el hierro tiene una velocidad de corrosión más alta, disminuyendo el ataque de los iones de cloro sobre las superficies conforme se amplía el período de exposición por la formación de capas protectoras de óxidos; otras investigaciones del acero $\mathrm{X} 65$ inmerso en 3,5\% de $\mathrm{NaCl}$ con tiempos de 1 y 24 horas, presenta la formación de óxidos producidos por la corrosión, los cuales reducen la corrosión uniforme y por picaduras [7,10]. La susceptibilidad de los aceros para tubería de línea X70, X80 y X100 expuestos a soluciones de $\mathrm{NaCl}$ al $3.5 \%$ con películas pasivas formadas por una solución de $0.5 \mathrm{~mol} / \mathrm{L} \mathrm{NaHCO}_{3}$, los aceros X100 demuestran que las velocidades de corrosión son menores y de la misma manera el comportamiento a la iniciación de picaduras que los aceros X80 y X70 [11]. Investigaciones en soldaduras de acero X70 indican que la ZAC tiene mayor disolución anódica en soluciones de pH neutral [12], el comportamiento es similar en soldaduras del acero X56 para tuberías de línea encontrando picaduras de mayor profundidad en la ZAC que el MB adyacente expuesto en agua de mar, [13]. El objetivo de este trabajo es estudiar el comportamiento a la corrosión de las soldaduras del acero API $5 \mathrm{~L} \mathrm{X70} \mathrm{unido}$ por el proceso DSAW a través de pruebas electroquímicas, inmersos en dos electrolitos, en $\mathrm{H}_{2} \mathrm{O}$ y $\mathrm{NaCl}$ al 3,5\%.

\section{Materiales y Métodos}

\subsection{Materiales y preparación de la muestras soldadas}

Durante el desarrollo experimental se utilizó un acero grado API 5L X70 para tubería de línea, el cual fue analizado microestructuralmente para corroborar las fases presentes. Con dicho análisis se corroboró que está constituido de ferrita y perlita, lo cual se puede apreciar en la Figura 1. Además se realizó un análisis para comprobar su composición química y el carbón equivalente (CE), la cual se muestra en la Tabla 1.

En la unión de los cupones de soldadura se utilizaron dos placas de acero cuyas dimensiones fueron de $152,4 \mathrm{~mm} \times 190,5 \mathrm{~mm} \times 12,7 \mathrm{~mm}$. La soldadura se realizó mediante el proceso de doble arco sumergido (DSAW) con un fundente granular y un electrodo de alambre sólido FA72 - EM13K de diámetro de 3,96 mm cuya composición química se muestra en la Tabla 2 y 3, es utilizado como sujeción externa zapatas en los cupones.

Tabla 1. Composición química del metal base API X-70 (\% en peso).

\begin{tabular}{ccccccccccccccc}
\hline Elemento & $\% \mathbf{C}$ & $\% \mathbf{S}$ & $\begin{array}{c}\% \\
\mathbf{M n}\end{array}$ & $\% \mathbf{P}$ & $\% \mathbf{S i}$ & $\% \mathbf{C r}$ & $\% \mathbf{N i}$ & $\begin{array}{c}\% \\
\mathbf{M o}\end{array}$ & $\% \mathbf{C u}$ & $\% \mathbf{V}$ & $\% \mathbf{N b}$ & $\% \mathrm{Ti}$ & $\% \mathbf{W}$ & $\mathbf{C E}$ \\
\hline IIw & \\
\hline
\end{tabular}


Tabla 2. Composición química del metal de aporte: fundente F7A2.

\begin{tabular}{|c|c|c|c|c|c|c|c|c|c|}
\hline Compuesto & $\% \mathrm{SiO}_{2}$ & $\%$ MnO & $\% \mathrm{MgO}$ & $\% \mathrm{CaF}_{2}$ & $\% \mathrm{Na}_{2} \mathrm{O}$ & $\% \mathrm{Al}_{2} \mathrm{O}_{3}$ & $\% \mathrm{TiO}_{2}$ & $\% F e O$ & $\begin{array}{l}\text { \% Metales } \\
\text { de aleación }\end{array}$ \\
\hline$\%$ Peso & 45 & 19 & 22 & 5 & 2 & 2 & 2 & 1 & 6 máx \\
\hline
\end{tabular}

Tabla 3. Composición química del metal de aporte: electrodo EM13K-H8.

\begin{tabular}{|c|c|c|c|c|c|c|c|c|c|c|c|}
\hline Elemento & $\% \mathrm{C}$ & $\%$ S & $\% M n$ & $\% \mathbf{P}$ & $\% \mathbf{S i}$ & $\% \mathrm{Cr}$ & $\% \mathrm{Ni}$ & $\%$ Mo & $\% \mathrm{Cu}$ & $\%$ V & Otros \\
\hline$\%$ Peso & $0,06-0,16$ & 0,030 & $0,90-1,40$ & 0,030 & $0,35-0,75$ & - & - & - & 0,35 & - & - \\
\hline
\end{tabular}

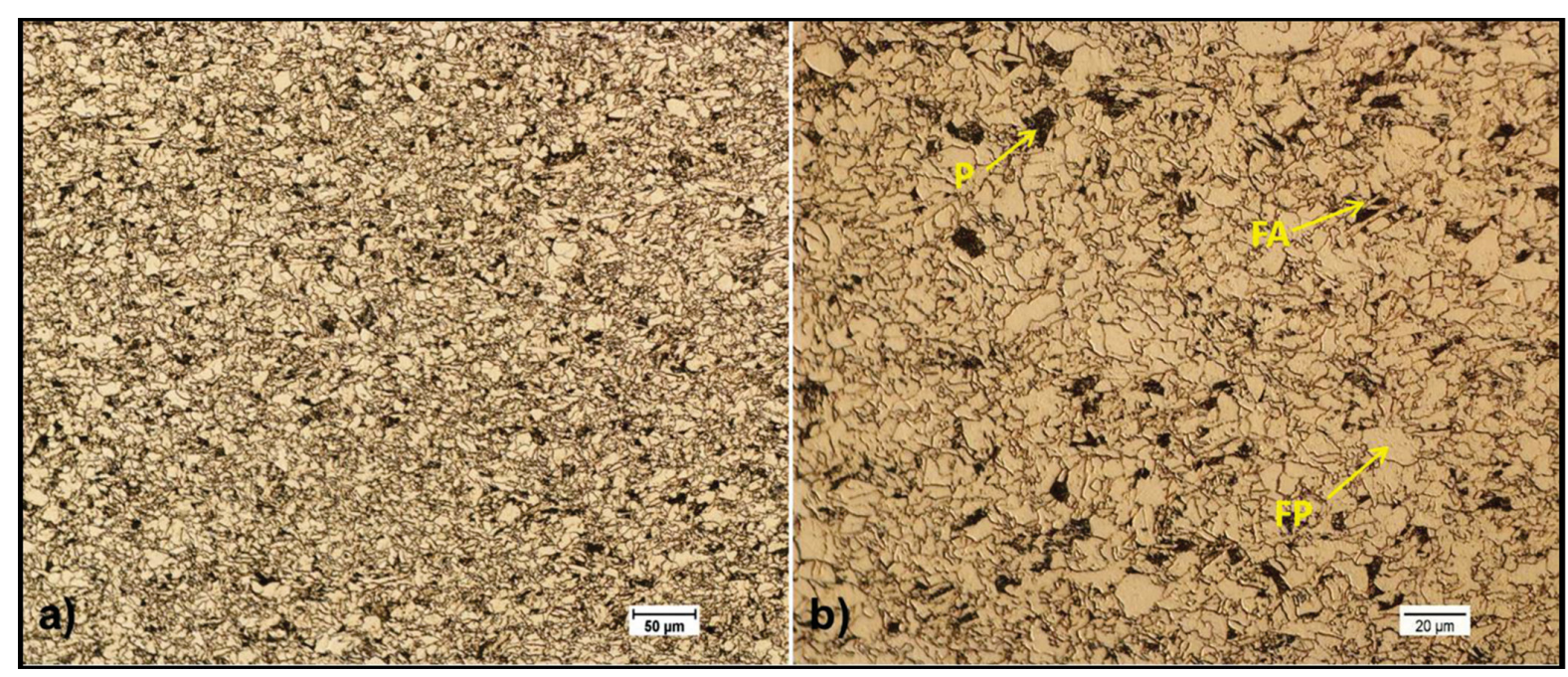

Figura 1. Microestructura del metal base API 5L X70 (FA: ferrita acicular; FP: ferrita poligonal; P: perlita). (a) 200X; (b) $500 \mathrm{X}$ atacado con Nital al $2 \%$.

Se realizó un biselado doble en $\mathrm{V}$, el interior de menor tamaño y el exterior mayor, tal como se observa en la Figura 2. Para unir los cupones de soldadura se variaron los parámetros de voltaje (v), amperaje (A) y velocidad de avance de acuerdo a los establecidos en la Tabla 4.

Tabla 4. Parámetros de soldadura empleados por el proceso DSAW en el acero API $5 \mathrm{~L}$ X70 (bisel interior y exterior).

\begin{tabular}{|c|c|c|c|c|c|c|}
\hline \multirow{2}{*}{$\begin{array}{l}\text { Número de } \\
\text { Muestra }\end{array}$} & \multicolumn{3}{|c|}{ Bisel interior } & \multicolumn{3}{|c|}{ Bisel exterior } \\
\hline & Voltaje (V) & $\begin{array}{l}\text { Amperaje } \\
\text { (A) }\end{array}$ & $\begin{array}{c}\text { Velocidad de } \\
\text { Avance (mm/s) }\end{array}$ & Voltaje (V) & $\begin{array}{l}\text { Amperaje } \\
\text { (A) }\end{array}$ & $\begin{array}{l}\text { Velocidad de } \\
\text { Avance }(\mathrm{mm} / \mathrm{s})\end{array}$ \\
\hline \multicolumn{7}{|l|}{1} \\
\hline \multicolumn{7}{|l|}{2} \\
\hline 3 & $29-31$ & $600-650$ & $13-16$ & $31-33$ & $650-725$ & $10-13$ \\
\hline \multicolumn{7}{|l|}{4} \\
\hline 5 & & & & & & \\
\hline
\end{tabular}

Se obtuvieron muestras para realizar las pruebas electroquímicas con dimensiones de $25 \mathrm{~mm} \times 7 \mathrm{~mm} \times 12,7 \mathrm{~mm}$ conectadas a un cable de cobre y recubiertas con resina en frío, el área de la superficie de exposición es de 1 cm², los especímenes antes de las pruebas son desbastados con lijas de papel de SiC desde los grados 320, 400 y 600, son lavados con agua destilada y alcohol siendo secados en aire caliente para evitar la oxidación. 
a)
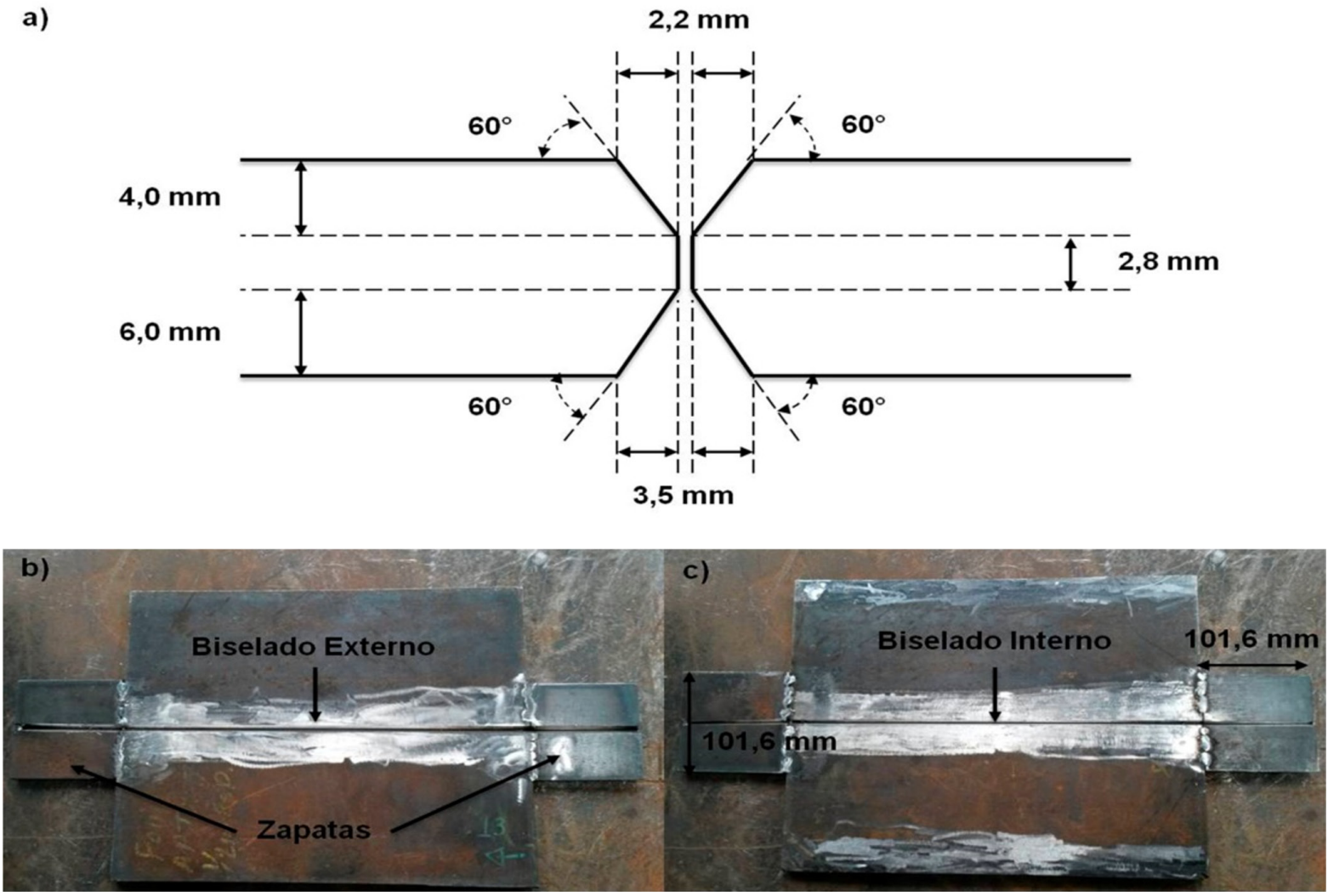

Figura 2. Diseño de los cupones de soldadura por DSAW: (a) dimensiones del biselado; cupones preparados para la experimentación; (b) biselado externo; (c) biselado interno.

\subsection{Pruebas electroquímicas}

Los electrodos de trabajo fueron desbastados hasta lija grado 600, enjuagados con agua destilada y secados con alcohol y aire caliente a presión. Los electrodos fueron guardados en un desecador por un tiempo de 24 horas.

Los electrolitos empleados en la investigación fueron: agua $\left(\mathrm{H}_{2} \mathrm{O}\right)$ y cloruro de sodio $(\mathrm{NaCl})$ al $3,5 \%$, ambos llevados a una temperatura ambiente.

Las pruebas electroquímicas se realizaron en un Potenciostato/Galvanostato/ZRA Gill AC de ACM Instruments. Se empleó una celda electrolítica de vidrio con una configuración de tres electrodos para las técnicas de ruido electroquímico $(R E)$ y curvas de polarización cíclica (CPC) como se muestra en la Figura 3. Se utilizó un alambre de Platino (Pt) como electrodo auxiliar (EA), electrodo de calomel saturado (ECS) como electrodo de referencia (ER) y los electrodos de trabajo (ET) fueron las soldaduras del acero API $5 \mathrm{~L} \mathrm{X70.}$

Con la técnica electroquímica de ruido electroquímico, se monitoreó la corriente y el potencial en función del tiempo, teniendo una adquisición de datos de 1.024 a una velocidad de 1 dato/s. Las pruebas de ruido electroquímico, fueron realizadas por inmersión en una celda de corrosión de 3 electrodos (dos electrodos de trabajo nominalmente idénticos), y un electrodo de referencia de calomel saturado [14].

Para las pruebas de curvas de polarización cíclica, se utilizó un arreglo de 3 electrodos: alambre de Pt como EA, ER un ECS, y soldaduras como ET. Los parámetros de experimentación fue polarizar el sistema desde $-500 \mathrm{mV}$ a $1.500 \mathrm{mV}$, con una velocidad de barrido de $60 \mathrm{mV} / \mathrm{min}$ y un ciclo completo [15].

\subsection{Análisis superficial de las muestras}

La apariencia superficial de cada una de las muestras fue observada bajo microscopía óptica en un equipo Nikon modelo SMZ - U y el análisis superficial de la corrosión producida fueron observadas por microscopía electrónica de barrido por electrones secundarios (SEM - SE) con el equipo HITACHI modelo S - 4800 para cada una de las regiones de las soldaduras como se observa en la macrografía de la Figura 4. 


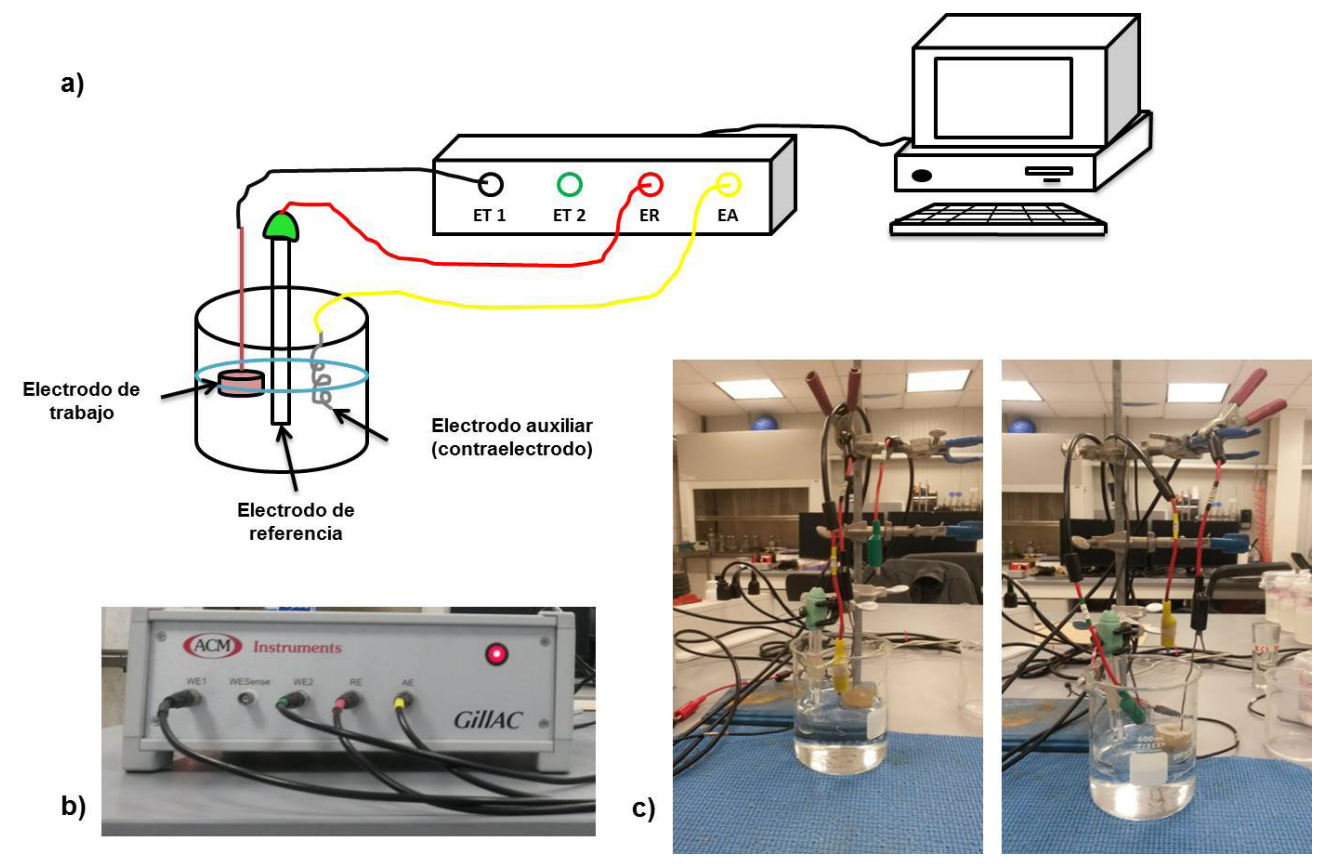

Figura 3. Representación esquemática de la prueba electroquímica: (a) equipo general empleado en las pruebas electroquímicas; (b) Galvanostato - Potenciostato-ZRA (GillAC); (c) celda electroquímica (lado izquierdo utilizando ET1, ER, EA; lado derecho utilizando ET1, ER, ET2).

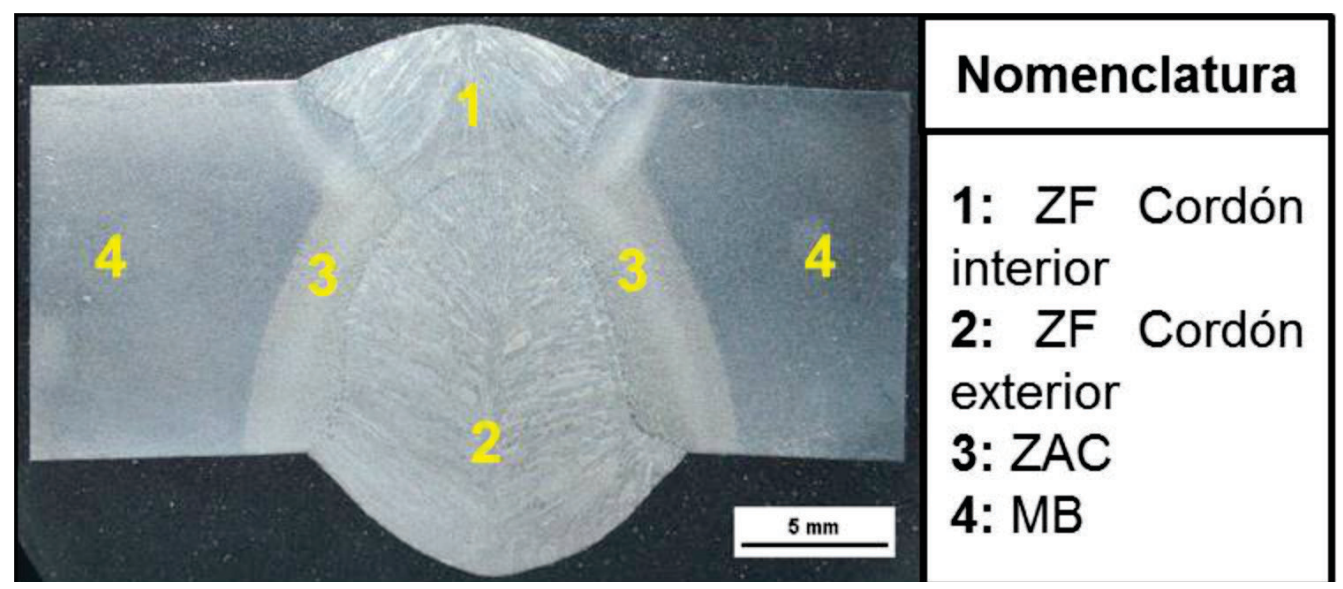

Figura 4. Macrografía de las soldaduras previas a las pruebas electroquímicas.

\section{Resultados y Discusión}

\subsection{Mediciones electroquímicas}

El comportamiento a la corrosión de las soldaduras y metal base del acero API X70 expuestos a distintos electrolitos: $\mathrm{H}_{2} \mathrm{O}$ y NaCl al 3,5\%, bajo la técnica de $\mathrm{RE}$ registran el $\mathrm{E}_{\text {corr }} \mathrm{i}_{\text {corr }}$, resistencia al ruido $\left(\mathrm{R}_{\mathrm{n}}\right)$, desviación estándar de la corriente $\left(\sigma_{\mathrm{i}}\right)$ y de potencial $\left(\sigma_{\mathrm{E}}\right)$, velocidad de corrosión (vel ${ }_{\text {corr }}$ ) e índice de localización (IL) presentados en la Tabla 5 y 6; a través de la técnica de CPC se obtienen potenciales de corrosión $\left(\mathrm{E}_{\text {corr }}\right)$, densidades de corrosión 
Comportamiento a la Corrosión del Acero API X70 Soldado por el Proceso de Doble Arco Sumergido Inmerso en Diferentes Medios Corrosivos

$\left(\mathrm{i}_{\text {corr }}\right)$, resistencia a la polarización $\left(\mathrm{R}_{\mathrm{p}}\right)$ para cada uno de los medios son presentados en la Tabla 7 y 8 . Las muestras son identificadas con una nomenclatura para el metal base en $\mathrm{H}_{2} \mathrm{O}$ como $\mathrm{MB}-\mathrm{AD}$ y en $\mathrm{NaCl}$ al 3,5\% como $\mathrm{MB}-\mathrm{S}$; y para las soldaduras expuestas en $\mathrm{H}_{2} \mathrm{O}$ son denominadas por $\mathrm{S}-\mathrm{ADX}$ y en $\mathrm{NaCl}$ al $3.5 \%$ por $\mathrm{S}-\mathrm{SX}$, la X indica el número de soldadura.

Tabla 5. Resultados de las pruebas electroquímicas de $\mathrm{RE}$ en $\mathrm{H}_{2} \mathrm{O}$.

\begin{tabular}{ccccccccc}
\hline Muestra & $\mathbf{E}_{\text {corr }}(\mathbf{m V})$ & $\boldsymbol{\sigma}_{\mathrm{E}}(\mathbf{m V})$ & $\boldsymbol{\sigma}_{\mathbf{i}}\left(\mathbf{m A} \mathbf{\mathbf { c m } ^ { 2 } )}\right.$ & $\mathbf{R}_{\mathbf{n}}\left(\mathbf{\Omega}^{*} \mathbf{c m}^{2}\right)$ & $\mathbf{i}_{\text {corr }}\left(\mathbf{m A} / \mathbf{c m}^{2}\right)$ & $\begin{array}{c}\mathbf{V e l}_{\text {corr }} \\
(\mathbf{m m} / \mathbf{a n ̃ o})\end{array}$ & $\mathbf{I L}$ & $\mathbf{T i p o}$ \\
MB-AD & $-360,64$ & 26,66 & $6,71 \mathrm{E}-03$ & $3.972,80$ & $6,57 \mathrm{E}-03$ & 0,08 & 0,11 & $\mathrm{~L}$ \\
S-AD1 & $-340,58$ & 30,24 & $7,07 \mathrm{E}-03$ & $4.279,40$ & $6,10 \mathrm{E}-03$ & 0,07 & 0,08 & $\mathrm{M}$ \\
S-AD2 & $-331,43$ & 20,66 & $2,71 \mathrm{E}-03$ & $7.617,80$ & $3,42 \mathrm{E}-03$ & 0,04 & 0,05 & $\mathrm{M}$ \\
S-AD3 & $-417,41$ & 18,19 & $2,74 \mathrm{E}-03$ & $6.636,80$ & $3,93 \mathrm{E}-03$ & 0,05 & 0,07 & $\mathrm{M}$ \\
S-AD4 & $-474,86$ & 11,59 & $1,62 \mathrm{E}-03$ & $7.160,10$ & $3,64 \mathrm{E}-03$ & 0,04 & 0,07 & $\mathrm{M}$ \\
S-AD5 & $-365,23$ & 22,67 & $3,72 \mathrm{E}-03$ & $6.091,60$ & $4,28 \mathrm{E}-03$ & 0,05 & 0,07 & $\mathrm{M}$ \\
\hline
\end{tabular}

Tabla 6. Resultados de las pruebas electroquímicas de RE en 3,5\% de $\mathrm{NaCl}$.

\begin{tabular}{ccccccccc}
\hline Muestra & $\mathbf{E}_{\text {corr }}(\mathbf{m V})$ & $\boldsymbol{\sigma}_{\mathbf{E}}(\mathbf{m V})$ & $\boldsymbol{\sigma}_{\mathbf{i}}\left(\mathbf{m A} / \mathbf{c m}^{2}\right)$ & $\mathbf{R}_{\mathbf{n}}\left(\mathbf{\Omega}^{*} \mathbf{c m}^{2}\right)$ & $\mathbf{i}_{\text {corr }}\left(\mathbf{m A} / \mathbf{c m}^{2}\right)$ & $\begin{array}{c}\mathbf{V e l}_{\text {corr }} \\
(\mathbf{m m} / \mathbf{a n ̃ o})\end{array}$ & IL & Tipo \\
MB-S & $-553,63$ & 7,63 & $4,21 \mathrm{E}-03$ & $1.811,2$ & $1,44 \mathrm{E}-02$ & 0,17 & 0,08 & $\mathrm{M}$ \\
S-S1 & $-562,78$ & 8,78 & $6,27 \mathrm{E}-04$ & 14.002 & $1,86 \mathrm{E}-03$ & 0,02 & 0,03 & $\mathrm{M}$ \\
S-S2 & $-603,96$ & 4,91 & $5,48 \mathrm{E}-03$ & 897,44 & $2,91 \mathrm{E}-02$ & 0,34 & 0,12 & $\mathrm{~L}$ \\
S-S3 & $-551,89$ & 9,75 & $5,85 \mathrm{E}-03$ & $1.668,2$ & $1,56 \mathrm{E}-02$ & 0,18 & 0,08 & $\mathrm{M}$ \\
S-S4 & $-562,91$ & 9,03 & $1,17 \mathrm{E}-03$ & $7.718,2$ & $3,38 \mathrm{E}-03$ & 0,04 & 0,04 & $\mathrm{M}$ \\
S-S5 & $-585,23$ & 5,97 & $3,19 \mathrm{E}-03$ & $1.871,9$ & $1,39 \mathrm{E}-02$ & 0,16 & 0,08 & $\mathrm{M}$ \\
\hline
\end{tabular}

Tabla 7. Resultados de las pruebas electroquímicas de $\mathrm{CPC}$ en $\mathrm{H}_{2} \mathrm{O}$.

\begin{tabular}{cccc}
\hline Muestra & $\mathbf{E}_{\text {corr }}(\mathbf{m V})$ & $\mathbf{R}_{\mathbf{p}}\left(\mathbf{\Omega}^{*} \mathbf{c m}^{2}\right)$ & $\mathbf{i}_{\text {corr }}\left(\mathbf{m A} / \mathbf{c m}^{2}\right)$ \\
MB-AD & $-481,42$ & 686,06 & $3,67 \mathrm{E}-02$ \\
S-AD1 & $-547,25$ & 626,94 & $4,02 \mathrm{E}-02$ \\
S-AD2 & $-516,49$ & 936,85 & $2,69 \mathrm{E}-02$ \\
S-AD3 & $-528,03$ & 810,39 & $3,11 \mathrm{E}-02$ \\
S-AD4 & $-535,22$ & $1.116,10$ & $2,26 \mathrm{E}-02$ \\
S-AD5 & $-520,92$ & 761,27 & $3,31 \mathrm{E}-02$ \\
\hline
\end{tabular}

Tabla 8. Resultados de las pruebas electroquímicas de CPC en 3,5\% de $\mathrm{NaCl}$.

\begin{tabular}{cccc}
\hline Muestra & $\mathbf{E}_{\text {corr }}(\mathbf{m V})$ & $\mathbf{R}_{\mathbf{p}}\left(\mathbf{\Omega}^{*} \mathbf{c m}^{2}\right)$ & $\mathbf{i}_{\text {corr }}\left(\mathbf{m A} / \mathbf{c m}^{2}\right)$ \\
MB-S & $-593,59$ & 4,43 & 5,69 \\
S-S1 & $-594,60$ & 4,03 & 6,25 \\
S-S2 & $-614,28$ & 5,00 & 5,04 \\
S-S3 & $-582,47$ & 4,88 & 5,16 \\
S-S4 & $-571,31$ & 4,22 & 5,97 \\
S-S5 & $-602,20$ & 3,54 & 7,11 \\
\hline
\end{tabular}

La susceptibilidad a la corrosión es eminente a ocurrir en ambos medios, sin embargo, el proceso de corrosión es más elevado en un medio salino, esto se observa en la vel corr del acero, mostrado en las Tablas 5 y 6, debido a la presencia del cloro tiende a acelerar la corrosión de los materiales [7]. El comportamiento de las soldaduras dentro de un medio de $\mathrm{H}_{2} \mathrm{O}$ en la muestra $\mathrm{S}-\mathrm{AD} 1$ es más elevada la velocidad de corrosión de 0,07 mm/año mientras que las soldaduras $S-A D 2$ y $S-A D 4$ tienen una vel ${ }_{\text {corr }}$ más baja de 0,04 mm/año. 
En el medio de $\mathrm{NaCl}$ al 3,5\% la muestra S - S2 tiene una vel ${ }_{\text {corr }}$ mayor de 0,34 mm/año y la más baja vel ${ }_{\text {corr }}$ en la muestra S - S1 siendo de 0,02 mm/año.

El análisis estadístico de las series de tiempo de potencia y corriente, permiten determinar el índice de localización (IL), haciendo referencia a Kelly, Inman y Hudson, el cálculo de la resistencia de ruido electroquímico $(R n)$, en la Ecuación 1 fue realizado por el cociente de la desviación estándar de las mediciones de ruido en potencial y corriente [16-19]:

$$
\mathrm{R}_{\mathrm{n}}=\frac{\sigma_{E}}{\sigma_{I}}
$$

$\sigma_{\mathrm{E}}$ : desviación estándar de ruido en potencial.

$\sigma_{1}$ : desviación estándar de ruido en la corriente.

$\mathrm{R}_{\mathrm{n}}$ : resistencia de ruido electroquímico.

El índice de localización en la Ecuación 2, es un parámetro que evalúa la variación de ruido en corriente, por medio de la razón de la desviación estándar en corriente y la raíz media cuadrática en corriente de acuerdo a [14,20]:

$$
\mathrm{IL}=\frac{\sigma_{i}}{I_{R M S}}
$$

IL: índice de localización.

$\sigma_{i}:$ desviación estándar de la corriente.

$\mathrm{I}_{\mathrm{RMS}}$ : raíz media cuadrática de la corriente.

A través del IL se determina el tipo de corrosión que ocurre dentro del sistema, definiendo los procesos de corrosión (localizada, uniforme o mixta) de acuerdo a los valores de la Tabla 9.

Tabla 9. Intervalos de valores de IL para identificar el tipo de corrosión en un sistema [20].

\begin{tabular}{ccc}
\hline & \multicolumn{2}{c}{ Valores de IL } \\
\cline { 2 - 3 } Tipo de Corrosión & Máx. & Mín. \\
\cline { 2 - 3 } & 1 & 0,1 \\
Localizada (L) & 0,1 & 0,01 \\
Mixta (M) & 0,01 & 0,001 \\
Uniforme (U) & & \\
\hline
\end{tabular}

Los resultados de las Tablas 5 y 6 indican que el tipo de corrosión es el mismo en ambos medios corrosivos con una corrosión mixta en la mayoría de las muestras de acuerdo a los valores de referencia en la Tabla 9, sin embargo, la muestra MB - AD en agua tiende a presentar una corrosión localizada de la misma manera para la muestra $\mathrm{S}-\mathrm{S} 2$ inmersa en $\mathrm{NaCl}$.

\subsection{Discusión de técnicas electroquímicas}

Con las técnicas electroquímicas se obtuvieron parámetros electroquímicos $\left(\mathrm{E}_{\text {corr }} \mathrm{I}_{\text {corr }}, \mathrm{R}_{\mathrm{n}}, \mathrm{IL}\right)$ que permiten obtener la velocidad y tipo de corrosión en las soldaduras en estudio. Con las series de tiempo de ruido en potencial y corriente se pudo observar mediante las fluctuaciones de la señal de ruido el comportamiento de la corrosión y con los valores de las desviaciones estándar de los datos en corriente y potencial se obtuvo la $R_{n}$ y con la desviación estándar de ruido en corriente y la RMS de la corriente se tuvo el IL. En las curvas de polarización cíclica se obtuvo mediante la $i_{\text {corr }}$ por medio de la extrapolación de las pendientes catódicas y anódicas de las curvas en un intervalo de $\pm 50 \mathrm{mV}$ a partir del $\mathrm{E}_{\text {corr }}$.

En la Figura 5 se observan las fluctuaciones de los transitorios de la corriente y potencial en las series de tiempo, para los materiales de estudio (metal base y soldaduras) expuestos en $\mathrm{H}_{2} \mathrm{O}$. La señal de ruido electroquímico en corriente no presenta grandes fluctuaciones, mismas que están entre 0,15 y $0,02 \mathrm{~mA} / \mathrm{cm}^{2}$, excepto las muestras $\mathrm{MB}-\mathrm{AD}$ y $\mathrm{S}-\mathrm{AD} 1$ exhiben una amplitud mayor pero sin transitorios que indiquen un tipo de corrosión localizada, 
Comportamiento a la Corrosión del Acero API X70 Soldado por el Proceso de Doble Arco Sumergido Inmerso en Diferentes Medios Corrosivos
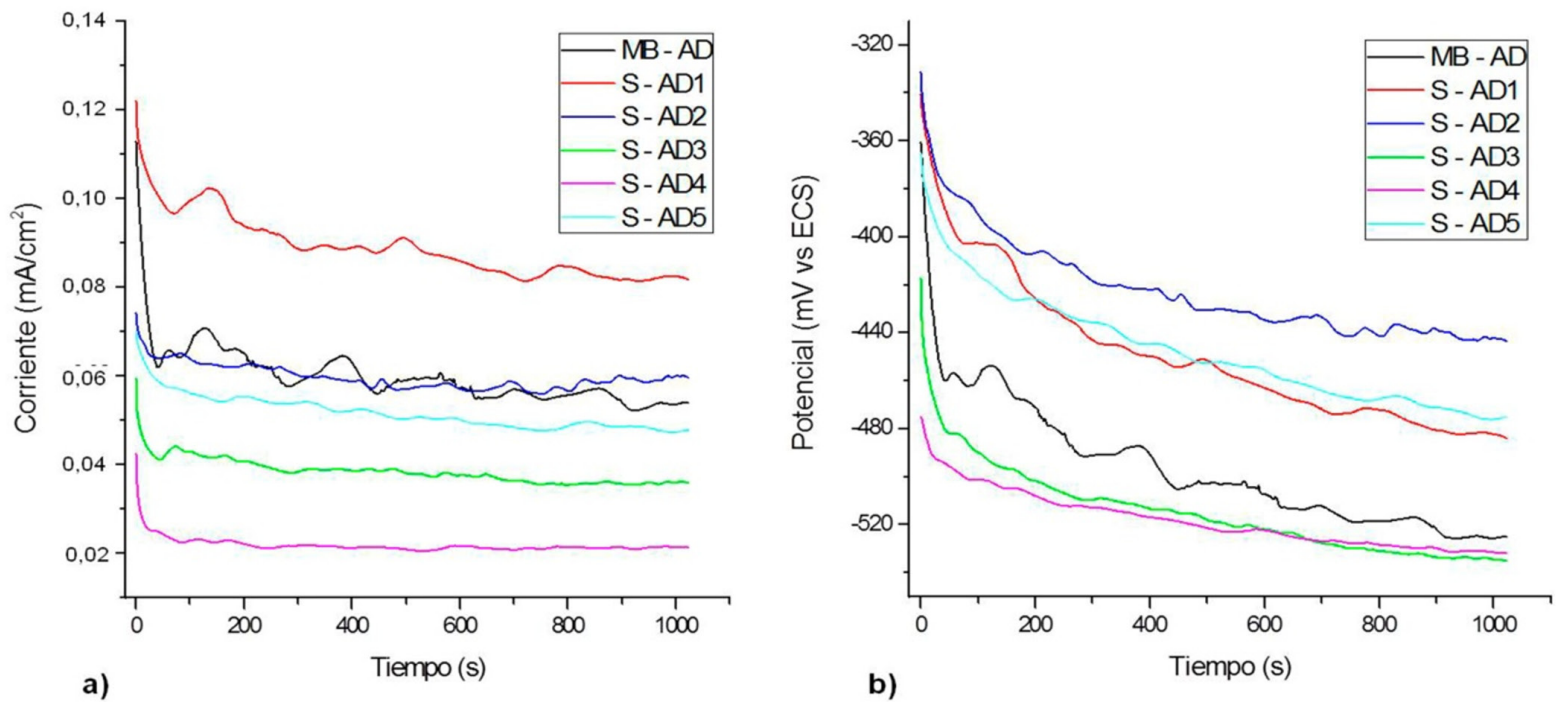

Figura 5. Series de tiempo de ruido electroquímico en corriente y potencial para las soldaduras del acero API $5 \mathrm{~L}$ X70 en $\mathrm{H}_{2} \mathrm{O}$.

sino más del tipo mixto [11]. Los potenciales de corrosión obtenidos del RE (Figura $5 b$ ) varían desde -330 a $-535 \mathrm{mV}$; las muestras $S-A D 3$ y $S-A D 4$ presentan potenciales más nobles que de la muestra MB - AD.

El comportamiento de la corrosión de las soldaduras y metal base, expuestas a medios salinos de 3,5\% se presenta en la Figura 6, donde se observa en las series de tiempo de ruido en corriente y potencial, que los valores de corriente están entre 0,02 y $0,09 \mathrm{~mA} / \mathrm{cm}^{2}$, dentro de esos rangos las muestras presentan una pequeña variabilidad en $\mathrm{S}-\mathrm{S} 1, \mathrm{~S}-\mathrm{S} 3, \mathrm{~S}-\mathrm{S} 5$ con respecto a la muestra $\mathrm{MB}-\mathrm{S}$; las fluctuaciones de corriente en la muestra S - S2 presentan perturbaciones cuya amplitud es evidente en la Figura 6a, esto indica que el tipo de corrosión es localizada contrario a las otras muestras con un tipo de corrosión mixto [11].

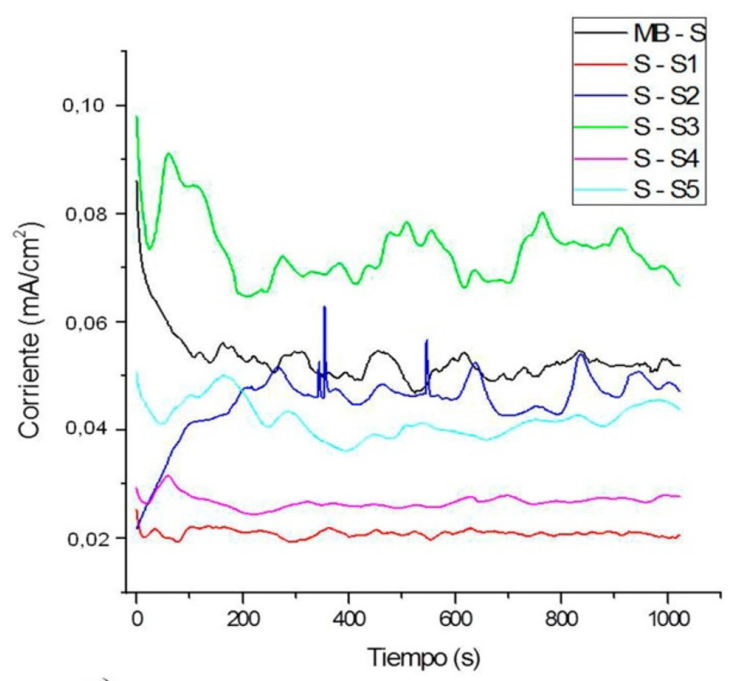

a)

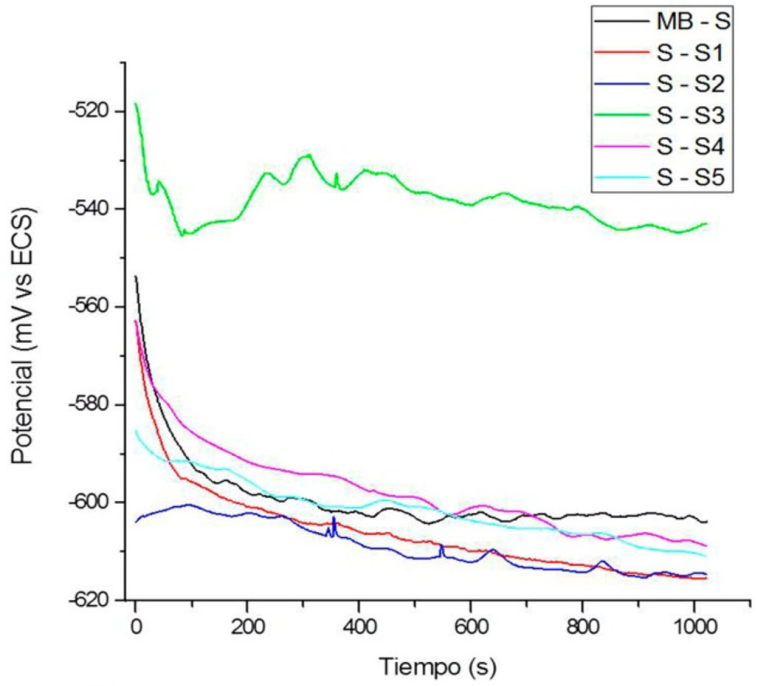

b)

Figura 6. Series de tiempo de ruido electroquímico en corriente y potencial para las soldaduras del acero API 5L X70 en $3,5 \%$ de $\mathrm{NaCl}$.

Las series de tiempo de ruido en potencial se pueden ver en la Figura $6 b$, donde se tiene pocas fluctuaciones del potencial con valores de -518 a $-615 \mathrm{mV}$. Sin embargo la muestra $\mathrm{S}-\mathrm{S} 3$ alcanza valores más nobles a los de $\mathrm{MB}-\mathrm{S}$. 
En distintos medios de corrosión el comportamiento electroquímico es variable, las corrientes y potenciales dentro de la prueba de RE van a ser mayores las corrientes alcanzadas en algunas soldaduras en $\mathrm{H}_{2} \mathrm{O}$ comparado en soluciones de $\mathrm{NaCl}$ al 3,5\%. Sin embargo los potenciales alcanzan valores más negativos para ambientes salinos que en agua, lo que indica que el sistema tiene tendencia a estar activo.

En la Figura 7 se presentan las curvas de polarización cíclica para el metal base y soldaduras del acero API 5L X70 expuestos a diferentes medios corrosivos, las mediciones muestran que los valores de potenciales disminuyen a valores más activos y la corriente aumenta al exponerse las muestras en soluciones de $\mathrm{NaCl}$ al 3,5\% teniendo en
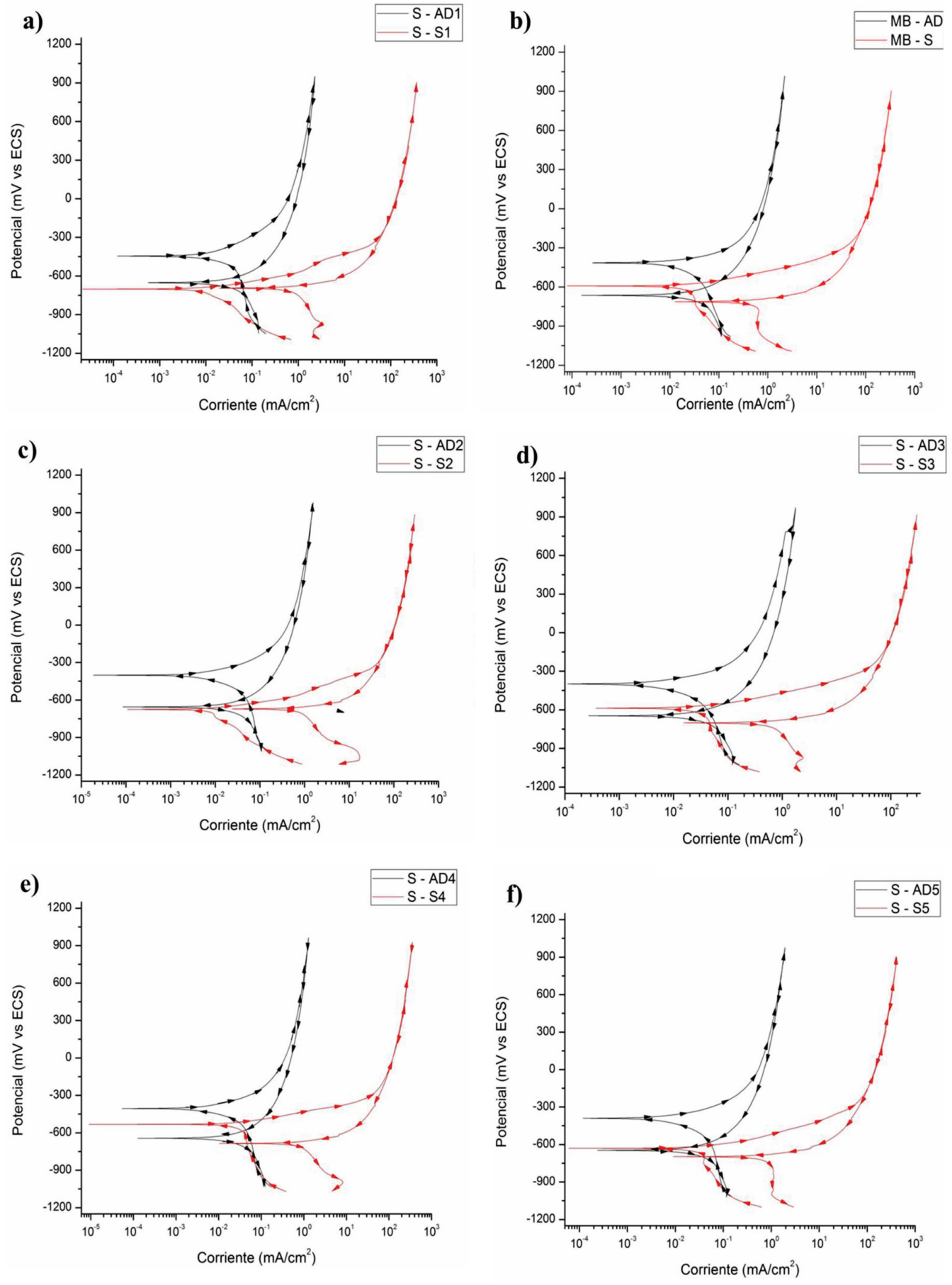

Figura 7. Curvas de polarización cíclica de soldaduras por DSAW de un acero API $5 \mathrm{~L} X 70$ inmerso en $\mathrm{H}_{2} \mathrm{O}$ y en $3,5 \%$ de $\mathrm{NaCl}$. 
Comportamiento a la Corrosión del Acero API X70 Soldado por el Proceso de Doble Arco Sumergido Inmerso en Diferentes Medios Corrosivos

la zona anódica regiones de activación más amplias [10]. El comportamiento de las soldaduras y el metal base en ambos medios tienen la tendencia de regresar ligeramente por dentro de la rama anódica (histéresis), exhibiendo un tipo de corrosión localizado y mixto.

Los valores de densidades de corriente, $\mathrm{i}_{\text {corr }}\left(\mathrm{mA} / \mathrm{cm}^{2}\right)$ en $\mathrm{H}_{2} \mathrm{O}$ son menores para las soldaduras con respecto al $M B-A D$ y los potenciales de corrosión en todas las muestras soldadas tienen resultados más negativos lo que indica que está más activo el sistema en corrosión. Los potenciales de corrosión en las soldaduras exhiben valores más negativos para $S-A D 1$ y menos en $S-A D 2$, las densidades de corriente $i_{\text {corr }}$ en la muestra $S-A D 5$ es mayor y en $\mathrm{S}-\mathrm{AD} 4$ con valores menores. $\mathrm{En} \mathrm{NaCl}$ al 3,5\% el $\mathrm{MB}-\mathrm{S}$ tiene densidades de corriente y potenciales de corrosión mayores que las soldaduras $\mathrm{S}-\mathrm{S} 3$ y $\mathrm{S}-\mathrm{S} 4$. Las densidades de corrosión y potenciales de corrosión en las soldaduras son mayores en la muestra $S-S 2$, con menores $i_{\text {corr }} y E_{\text {corr }}$ para $S-S 3$ y $S-S 4$ respectivamente.

\subsection{Análisis superficial}

Después de realizar las pruebas electroquímicas, se observaron superficialmente mediante MEB para analizar el efecto de los medios corrosivos. En la Figura 8a, b el metal base exhibe cantidades elevadas de picaduras distribuidas aleatoriamente y concentradas en algunas zonas; en la Figura 8c, d la exposición del metal base en $\mathrm{NaCl}$ remueve una capa homogénea sobre la superficie del acero X70 además de exhibir picaduras de mayor profundidad pero en menor cantidad.

Todas las muestras de soldadura son corroídas en ambos medios, en la Figura 9a-d se muestra la soldadura con doble cordón expuesto en $\mathrm{H}_{2} \mathrm{O}$; el cordón interior (Figura 9a) tiende a corroer por picaduras concentradas en altas proporciones, la interfase entre el cordón interior y la ZAC (Figura 9b) presenta también picaduras de forma

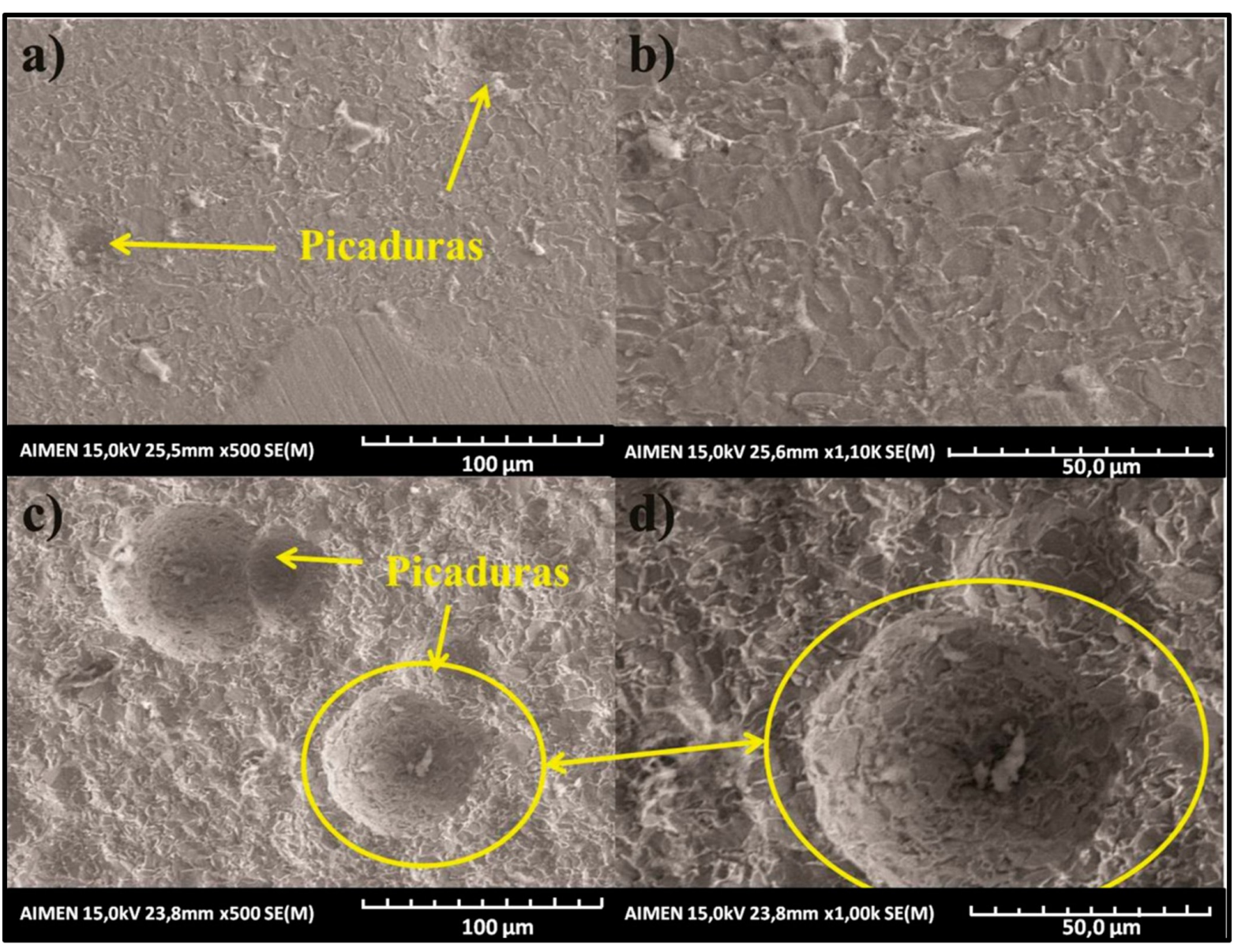

Figura 8. Morfología de la corrosión superficial del acero API $5 \mathrm{~L}$ X70 después de las pruebas electroquímicas: $(a, b)$ inmerso en $\mathrm{H}_{2} \mathrm{O}$; (c, d) inmerso en $3,5 \%$. de $\mathrm{NaCl}$. 


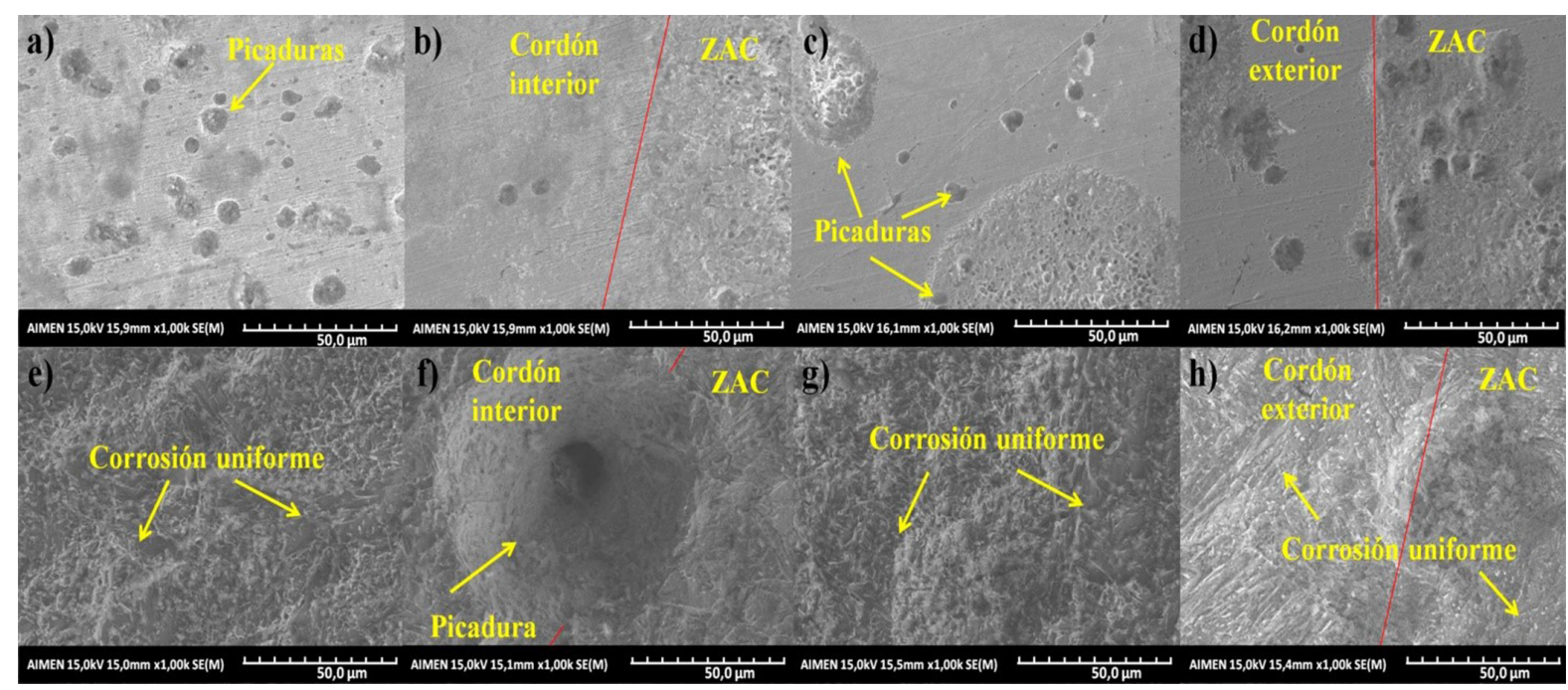

Figura 9. Morfología de la corrosión superficial de las soldaduras del acero API 5L X70 después de las pruebas electroquímicas: (a) cordón interior; (b) interfase del cordón interior y ZAC; (c) cordón exterior; (d) interfase del cordón exterior y ZAC inmersos en $\mathrm{H}_{2} \mathrm{O}$; (e) cordón interior; (f) interfase del cordón interior y ZAC; (g) cordón exterior; (h) interfase del cordón exterior y ZAC inmerso en 3,5\% de $\mathrm{NaCl}$.

aleatoria. La Figura 8e-h expone las soldaduras en 3,5\% de $\mathrm{NaCl}$ en ambos cordones e interfases (cordones y ZAC) corroídos por un proceso del tipo mixto debido al medio que degrada las superficies de las soldaduras y presentan picaduras más profundas específicamente en la ZAC.

Mediante microscopía óptica en la Figura 10, se observan las morfologías de las picaduras en el metal base en mayor cantidad concentradas y distribuidas aleatoriamente expuestos en $\mathrm{H}_{2} \mathrm{O}$ (Figura 9a), contrario en $\mathrm{NaCl}$ la muestra es corroída uniformemente y pocas picaduras son visibles (Figura 9b); las soldaduras exhiben también una corrosión mixta por la concentración y aislamiento de picaduras en soluciones de $\mathrm{H}_{2} \mathrm{O}$ (Figura 9c, d); las soldaduras en $\mathrm{NaCl}$ son corroídas de forma mixta (Figura 9e, f) igual que el MB.

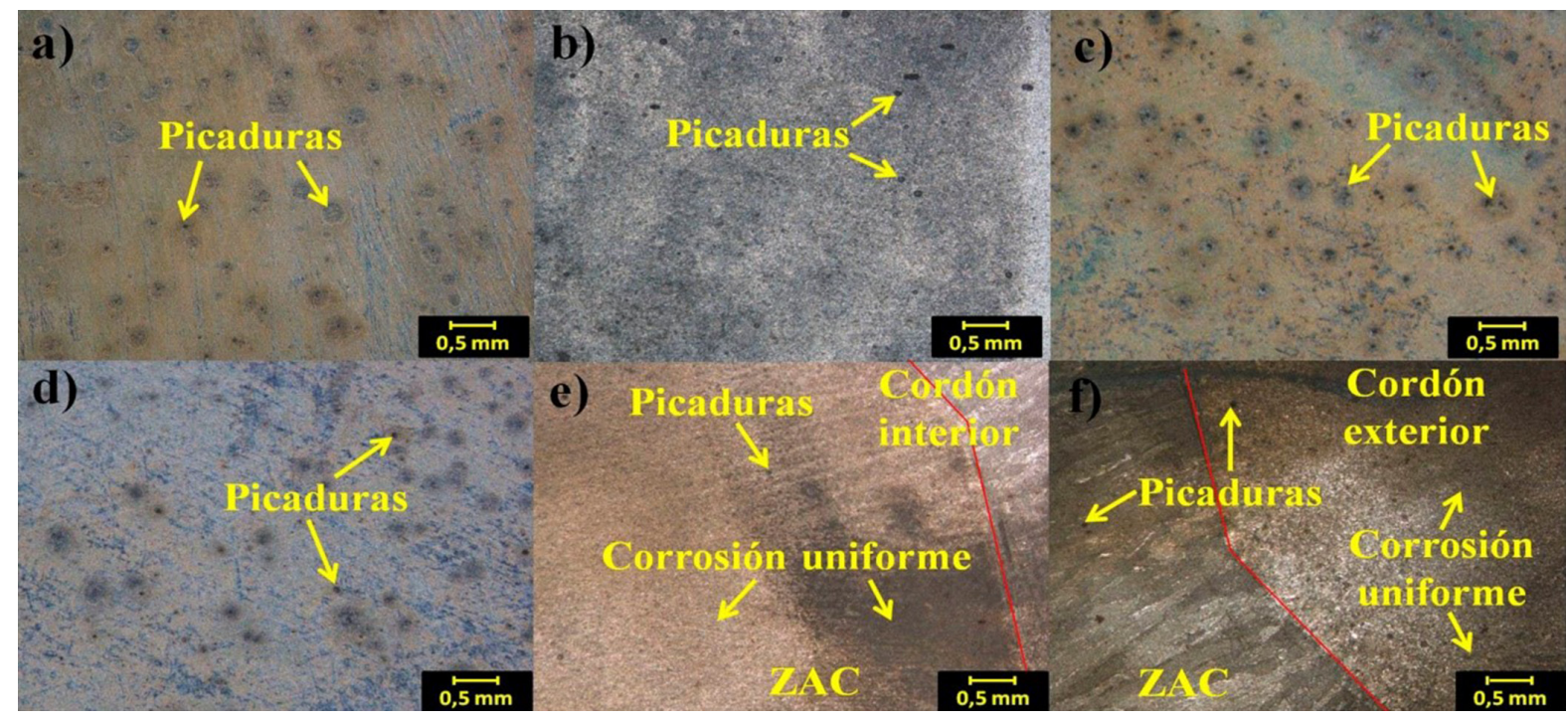

Figura 10. Micrografías del acero API $5 \mathrm{~L}$ X70 en soluciones corrosivas: (a) $\mathrm{MB}$ en $\mathrm{H}_{2} \mathrm{O}$; (b) $\mathrm{MB}$ en $\mathrm{NaCl}$ al 3,5\%; (c) cordon interior en $\mathrm{H}_{2} \mathrm{O}$; (d) cordon exterior en $\mathrm{H}_{2} \mathrm{O}$; (e) interfase (cordon interior y ZAC) en $\mathrm{H}_{2} \mathrm{O}$; (f) interfase (cordon exterior y ZAC) en 3,5\% de $\mathrm{NaCl}$. 
Comportamiento a la Corrosión del Acero API X70 Soldado por el Proceso de Doble Arco Sumergido Inmerso en

\section{Conclusiones}

- La susceptibilidad a la corrosión del acero API 5L X70 y las soldaduras en diferentes electrolitos es eminente a ocurrir.

- El comportamiento a la corrosión de las soldaduras y metal base inmersos en $\mathrm{H}_{2} \mathrm{O}$ y en 3,5\% de $\mathrm{NaCl}$, indican un tipo de corrosión mixta debido a la presencia de picaduras concentradas en zonas y de manera aleatoria con excepción de las muestras $\mathrm{MB}-\mathrm{AD}$ y S - S2 indican un tipo de corrosión localizado, determinado por el IL, CPC.

- Con la caracterización por MEB, se observa un medio de exposición más agresivo en el que presenta contenidos de cloro, provocando remoción del material de forma superficial, bajo un proceso de corrosión uniforme e inclusive zonas con picaduras tanto en los cordones de soldadura, interfase y metal base.

- Las probetas son corroídas en los cordones de soldadura y metal base en $\mathrm{H}_{2} \mathrm{O}$ por picaduras y en $\mathrm{NaCl}$ exhiben remoción superficial por corrosión en las soldaduras además de picaduras concentradas en la interfase y cordones de soldadura debido a que el acero API 5L X70 es un material susceptible a la corrosión no exhibe pasivación superficial acelerando el proceso de corrosión en un medio más agresivo.

- Las velocidades de corrosión incrementan al exponer el acero y soldaduras a un medio más agresivo, S - AD1 es más elevada la vel corr de $0,07 \mathrm{~mm} / \mathrm{año}$ en $\mathrm{H}_{2} \mathrm{O}$ y $\mathrm{NaCl}$ al 3,5\% la muestra $\mathrm{S}-\mathrm{S} 2$ tiene una vel corr mayor de 0,34 mm/año, se puede concluir que en la muestra S - AD4 presenta mejor comportamiento a la corrosión con $0,04 \mathrm{~mm} / a$ ño y $0,04 \mathrm{~mm} /$ año respectivamente.

\section{Agradecimientos}

Los autores expresan sinceramente agradecimientos a los colaboradores para el desarrollo de este trabajo realizado en la Corporación Mexicana de Investigación en Materiales S.A. de C.V. de Saltillo Coahuila en colaboración con el Centro de Investigación e Innovación en Ingeniería Aeronáutica de la FIME Nuevo León y el apoyo externo del equipo perteneciente a la Asociación de Investigación Metalúrgica del Noroeste de Porriño, Pontevedra, España.

\section{Referencias}

[1] ASM International Committee. Carbon and low: alloy steels. In: ASM Handbook Commite. ASM Handbook properties and selection: irons, steels, and high performance alloys. Ohio: ASM International; 1990. vol. 1-2, p. 262-962.

[2] Smallman RE, Ngan AHW. New materials. In: Tooley M. Design engineering manual. |Amsterdam: Elsevier; 2010. p. 209-210.

[3] Winsor FJ. High strengh low alloy (HSLA) structural steels. In: ASM Handbook Commite. ASM Handbook Welding, brazing and soldering. Ohio: ASM International; 1993. vol. 6, p. 539-1627.

[4] American Petroleum Institute - API. API 5L: specification for line pipe. 43. ed. Washington: API; 2004. p. 1-5.

[5] Jeffus LF. Introduction to welding. In: Jeffus LF. Welding principles and applications. 5. ed. New York: Delmar Learning; 2004. vol. 1, p. 338-339.

[6] Swain RA, O'Brien A. Submerged arc welding. In: Welding Handbook Committee. Welding handbook. 9. ed. Miami: American Welding Society; 2004. vol. 2, 6, p. 256-267.

[7] Sherif el SM. A comparative study on the electrochemical corrosion behavior of iron and X-65 steel in $4.0 \mathrm{wt} \%$ sodium chloride solution after different exposure intervals. Molecules (Basel, Switzerland). 2014;19(7):9962-9974. http://dx.doi. org/10.3390/molecules19079962. PMid:25010468.
[8] Stansbury EE, Buchanan RA. Fundamentals of electrochemical corrosion. Ohio: ASM International; 2000. p. 1- 21.

[9] Kelly RG. Electrochemical techniques in corrosion science and engineeringk. New York: Marcel Dekker; 2003. p. 95-96.

[10] Sherif ELS, Almajid AA, Khalil KA, Junaedi H, Latief FH. Electrochemical studies on the corrosion behavior of API X65 pipeline steel in chloride solutions. International Journal of Electrochemical Science. 2013;8:9360-9370.

[11] Zhao Y, Liang P, Shi Y, Zhang Y, Yang TAO. The pitting susceptibility investigation of passive films formed on $\mathrm{X} 70, \mathrm{X} 80$, and $\mathrm{X} 100$ pipeline steels by electrochemical noise and Mott-Schottky measurements. International Journal of Corrosion. 2015;2015:110. http://dx.doi.org/10.1155/2015/298584.

[12] Zhang GA, Cheng YF. Micro-electrochemical characterization of corrosion of welded X70 pipelinesteel in near-neutral pH solution. Corrosion Science. 2009;51(8):1714-1724. http:// dx.doi.org/10.1016/j.corsci.2009.04.030.

[13] Chaves IA, Melchers RE. Pitting corrosion in pipeline steel weld zones. Corrosion Science. 2011;53(12):4026-4032. http://dx.doi. org/10.1016/j.corsci.2011.08.005.

[14] American Society for Testing Materials - ASTM. ASTM G19909: standard guide for electrochemical noise measurement. Ohio: ASTM; 2009. p. 1-9. 
[15] American Society for Testing Materials - ASTM. ASTM G5-13: standard reference test method for making potentiodynamic anodic polarization measurments. Ohio: ASTM; 2013. p. 1-9.

[16] Cottis PA, Turgoose S. Electrochemical impedance and noise. Houston: NACE International; 1999. (Corrosion Testing Made Easy Series, 7).

[17] Barragán F, Guardián R, Menchaca C, Rosales I, Uruchurtu J. Electrochemical corrosion of hot pressing titanium coated steels for biomaterial applications. International Journal of Electrochemical Science. 2010;5(12):1799-1809.
[18] Legat A, Dolecek V. Chaotic analysis of electrochemical noise measured on stainless steel. Journal of the Electrochemical Society. 1995;6(6):1851. http://dx.doi.org/10.1149/1.2044205.

[19] Sanchez Amaya JM, Cottis RA, Botana FJ. Shot noise and statistical parameters for the estimation of corrosion mechanisms. Corrosion Science. 2005;47(12):3280-3299. http://dx.doi.org/10.1016/j. corsci.2005.05.047.

[20] Kelly KG, Inman ME, Hudson JL. Electrochemical noise measurement for corrosion applications. ASTM Special Technical Publication. 1996;1277:34-49. 